\title{
Semantic interference in the picture-word interference task: Is there a pre-lexical, conceptual contribution to the effect?
}

\author{
Jörg D. Jescheniak ${ }^{1}$. Stefan Wöhner ${ }^{1} \cdot$ Hanna S. Bethcke ${ }^{1} \cdot$ Marie C. Beaupain ${ }^{1}$
}

Published online: 2 January 2020

(C) The Psychonomic Society, Inc. 2020

\begin{abstract}
Picture naming takes longer in the presence of a semantic-categorically related distractor word compared to an unrelated distractor word. This semantic interference effect in the picture-word interference (PWI) task is an empirical cornerstone in speech production research and of central importance in theory development and evaluation. Prominent models locate the effect at an abstract lexical level, yet only few studies have tested for a possible pre-lexical, conceptual contribution. Moreover, those studies that did are not conclusive. We re-explored the locus of semantic interference by contrasting two task versions that were implemented in as parallel a fashion as possible, but differed with respect to the processing stages involved: naming pictures (requiring conceptual processing and lexical processing) and deciding on their natural size (requiring conceptual processing only). We predicted semantic interference in naming, replicating the standard effect. If part of the effect is localized at the conceptual level, we predicted interference in size decision, too. We found semantic effects in both tasks but with different polarity - interference in naming and facilitation in size decision. This pattern supports the view that semantic interference in PWI has its locus at the lexical level and its origin at the conceptual level.
\end{abstract}

Keywords Speech production $\cdot$ Semantic interference $\cdot$ Picture-word interference task

\section{Introduction}

Picture naming takes longer in the presence of a semantically related distractor word compared to an unrelated distractor word (Glaser \& Düngelhoff, 1984; Jescheniak, Schriefers, \& Hantsch, 2003; La Heij, 1988; Lupker, 1979; Roelofs, 1992; Schriefers, Meyer, \& Levelt, 1990). This semantic interference effect is an empirical cornerstone in speech production research and central for the evaluation of contemporary models (Levelt, Roelofs, \& Meyer, 1999; Roelofs, 1992, 2018; Starreveld \& La Heij, 1995). Prominent models assume that its locus is at the lexical level and that it reflects a competitive selection mechanism by which the (lexical) target

Electronic supplementary material The online version of this article (https://doi.org/10.3758/s13423-019-01667-w ) contains supplementary material, which is available to authorized users.

Jörg D. Jescheniak

jdj@uni-leipzig.de

1 Institute of Psychology - Wilhelm Wundt, Leipzig University, Neumarkt 9-19, D-04109 Leipzig, Germany representation is selected among a set of co-activated (lexical) candidates (e.g., Abdel Rahman \& Melinger, 2009; Damian \& Bowers, 2003; Levelt et al., 1999; Roelofs, 1992; Schriefers et al., 1990). This assumption, however, is not trivial. Speech production starts with conceptual preparation, that is the identification of a lexical concept (Levelt et al., 1999) or a set of semantic features (Dell, Martin, \& Schwartz, 2007; Oppenheim, Dell, \& Schwartz, 2010), which then drives the retrieval of a word at the lexical level. As the conceptual level is the "semantic database" which "brings meaning to all verbal and non-verbal stimuli" (Lambon Ralph, 2014, p. 1), and speech production models also assume detailed semantic information to be stored at this level (Roelofs, 1992; Levelt et al., 1999; Oppenheim et al., 2010), it appears a natural candidate locus of semantic interference. If one assumes a selection mechanism at the conceptual level (e.g., Bloem \& La Heij, 2003; Navarrete, Del Prato, Peressotti, \& Mahon, 2014) with similar properties as implemented at the lexical level in competitive lexical selection models, part of the semantic interference effect could well be located at that level. However, many researchers view the conceptual level as the locus of semantic facilitation due to priming (Abdel Rahman \& Melinger, 2009; Collins \& Loftus, 1975; Finkbeiner \& 
Caramazza, 2006; Levelt et al., 1999; Navarrete \& Costa, 2005; Roelofs, 1992), and some propose that semantic interference in PWI indeed reflects the net effect of facilitation during conceptual processing (due to priming from the distractor to the target and vice versa) and interference during lexical processing (due to competitive selection; Abdel Rahman \& Melinger, 2009; Hantsch, Jescheniak, \& Schriefers, 2009; Navarrete \& Costa, 2005).

These accounts make different predictions with respect to the effect of semantically related versus unrelated distractor words during conceptual versus lexical processing of target pictures. If semantic interference results from a selection process located at the lexical (or any later) level, no interference or possibly even facilitation should be obtained in tasks requiring conceptual but no lexical processing. In contrast, if conceptual processing involves a similar selection process to that proposed for lexical processing, interference should also be obtained in tasks requiring conceptual processing only.

So far, only few studies directly tested for a conceptual versus a lexical locus of semantic interference in PWI, with inconclusive results. In one study, Lupker and Katz (1981) asked participants to classify whether a target picture depicted a dog. The authors observed semantic interference from written distractor words and concluded that it is localized at the decision stage (i.e., conceptual level), not the verbal response preparation stage (i.e., lexical and later levels). However, participants possibly based their decision on checking whether a picture can be named as "dog" (via implicit naming). If so, the effect would also be compatible with a lexical locus interpretation (Damian \& Bowers, 2003). In another study, Schriefers et al. (1990) traced the locus of semantic interference by varying the task. In picture naming, semantic interference was observed with spoken distractor words. In old-new picture classification (new experimental pictures), there was no effect. The absence in the non-lexical task led the authors to conclude that semantic interference is localized at the lexical not the conceptual level. Critically, this conclusion rests on the assumption that the recognition task demanded sufficiently detailed semantic processing. However, if the set of old pictures is small and perceptual differences between pictures are large, participants could base their responses (partly) on shallow perceptual processing, reducing the chance of observing a semantic effect (Meyer, 1996). In yet another study, Damian and Bowers (2003) kept the task constant but varied the distractor type. With visual distractor words, semantic interference was obtained in picture naming. With distractor pictures, it disappeared. From its absence with non-lexical distractors the authors concluded that semantic interference is localized at the lexical, not the conceptual, level (see also Navarrete \& Costa, 2005; Roelofs, 2008). This conclusion presupposes that distractor pictures are not lexically encoded. However, later studies showed phonological facilitation (Morsella \& Miozzo, 2002; Roelofs, 2008; cf. Jescheniak et al., 2009) and semantic interference with distractor pictures (Mädebach, Wöhner, \& Jescheniak, 2018b; see also
Jescheniak, Matushanskaya, Mädebach, \& Müller, 2014; Matushanskaya, Mädebach, Müller, \& Jescheniak, 2016), questioning the generalizability of Damian and Bower's finding.

Using a dual task approach, Dell'Acqua, Job, Peressotti, and Pascali (2007) found semantic interference from distractor words to be attenuated if the picture naming task followed a tone discrimination task at short task SOA. This pattern suggested that the effect is localized prior to central processing (viz. lexical selection). However, later studies failed to replicate this pattern (see Piai, Roelofs, \& Schriefers, 2014).

Using a dual task approach, Dell'Acqua, Job, Peressotti, and Pascali (2007) found semantic interference from distractor words to be attenuated if the picture naming task followed a tone discrimination task at short task SOA. This pattern suggested that the effect is localized prior to central processing (i.e., prior to lexical selection). However, later studies failed to replicate this pattern (see Piai, Roelofs, \& Schriefers, 2014).

In the light of these inconclusive findings and given the theoretical importance of semantic interference in PWI, it seems important to re-reconsider whether its locus is only at the lexical level or whether there are contributions from the conceptual level, too. We addressed this issue by investigating the effect in two tasks differing in the processing stages involved. In picture naming - requiring conceptual processing and lexical processing, participants named the objects. In size decision requiring conceptual but no lexical processing, participants classified them according to their natural size. If the locus of semantic interference is both at the conceptual and the lexical levels, interference should be obtained in naming and in size decision, albeit attenuated in size decision. If, instead, the locus of the effect is only at the lexical level, no interference or possibly even facilitation should be found in size decision.

We implemented the tasks in as parallel a fashion as possible, including type of design, materials, experimental lists, and familiarization procedure. Participants were assigned to tasks alternatingly. We used many items and a non-lexical task that required detailed semantic processing because size information is only available upon successful conceptual identification of the depicted objects.

We report all data exclusions, manipulations, and measures implemented. We preregistered our study at: https:// aspredicted.org/s557m.pdf. All materials, data, and analysis scripts are available at: https://osf.io/rt7sy/.

\section{Method}

\section{Participants}

Sixty-four native speakers of German participated (52 females, average age 22.97 years). Half performed the size decision task, and half performed the picture naming task. Task assignment was altered after any two participants. Participants 
received course credit or $8 €$. One participant was replaced (> $20 \%$ errors during practice).

\section{Materials}

Experimental pictures were 40 black line drawings of objects (max. $8.4 \times 8.4^{\circ}$ at 60 -cm viewing distance). Half of the objects had a natural size smaller than a selected standard (a box visible at the beginning of the experiment, $26.5 \times 18.0 \times 12.5 \mathrm{~cm}$ ), the other half larger than it. For each picture, a semantic-categorically related word was selected as distractor; it denoted an object of the same size class. To create the unrelated distractor condition, distractors were reassigned to pictures denoting objects of the same size class. We added 40 filler pictures (half depicting small objects and half depicting large objects) and distractor words for which the size of depicted objects and of objects denoted by the (related and unrelated) distractors differed. ${ }^{1}$ Eight additional pictures and corresponding distractor words were selected for creating practice and warm-up trials. For a complete list of the materials see https://osf.io/rt7sy/.

Distractor words were superimposed onto the pictures embedded in a rectangle of a light-gray background color (RGB 220220 220). The rectangle's size was fixed for a given picture but varied between pictures (width determined so that it just fitted both the related and the unrelated distractor word). Distractors were printed in Arial bold $\left(1.7 \times 3.1-8.7^{\circ}\right)$.

\section{Design}

Independent variables were distractor condition (semantically related vs. unrelated) and task (naming vs. size decision). Distractor condition was tested within participants and items, task within items but between participants. Dependent variables were response latencies and error rates.

We created two blocks of trials. In each block, each of the 40 experimental and 40 filler items appeared once, half of each type with related distractors and half with unrelated distractors. The sequence of distractor conditions per item was counterbalanced

\footnotetext{
${ }^{1}$ Semantically closely related objects tend to share features, including size. Because our study crucially depended on the demonstration of a sizeable interference effect in naming (requiring the selection of semantically closely related picture-distractor pairs), we restricted experimental items to pictures with size-congruent distractors and added (semantically less closely related) filler items and distractors (depicting and denoting objects differing in size). Overall, depicted and denoted objects were of the same size in half of the trials and of a different size in the other half, while for each item, related and unrelated distractors denoted similar-sized objects. Our construction of the material set might thus have facilitated the processing of the experimental items (with size-congruent related and unrelated distractors) compared to the processing of the filler items (with size-incongruent related and unrelated distractors, see Hantsch, Jescheniak, \& Schriefers, 2005; Hantsch et al., 2009; Kuipers \& La Heij, 2008). Critically, however, size congruency cannot have had any impact on the critical difference between related and unrelated distractor conditions (because it was held constant for each individual item in each item set).
}

in two parallel lists (i.e., lists with the same sequence of target pictures, but different sequences of distractor words). Sixteen pseudo-randomizations of these list pairs resulted in 32 lists overall. Each list was used once for the naming task and once for the size decision task. Lists were created using Mix (Van Casteren \& Davis, 2006), with the constraints that picture or distractor repetitions were separated by a minimum of eight trials, the same distractor condition or object size was not repeated in more than four consecutive trials, and semantically related pictures and pictures or distractor words with the same initial phoneme did not appear in consecutive trials.

\section{Procedure and apparatus}

First, participants received written instructions and a booklet. Participants were either instructed to name the object or to decide whether it is smaller or larger than the box visible during familiarization and practice (left button - smaller, right button - larger) as quickly as possible. Depending on the task, either the object's name or size was printed below each picture in the booklet. Two practice blocks followed. First, the task was introduced; each picture was responded to once (88 trials). Erroneous responses were corrected. Next, distractor words were introduced (eight practice trials); participants were instructed to ignore the words. Then, the two blocks with 40 experimental and 40 filler trials each were presented (two warm-up trials at the beginning of each block, blocks separated by a pause).

The experiment was controlled by NESU (Nijmegen Experimental Setup). Pictures and words were presented simultaneously for $800 \mathrm{~ms}$ on a TFT-monitor. Responses were registered from picture onset with a microphone connected to a hardware voice-key or with a push-button box (max. $2.5 \mathrm{~s}$ ).

\section{Results}

Analyses were restricted to the experimental trials; for additional exploratory analyses on all trials (including filler trials) and filler trials only see the Supplementary Material. For the reaction-time (RT) analyses, we discarded trials with (a) missing or erroneous response, (b) disfluent response or participant's non-speech sound triggering the voice key, (c) voicekey malfunction, or (d) latency $<150 \mathrm{~ms}$ (4.22\%). Cases (a) and (b) were included in the error analyses (2.29\%). Analyses were conducted in R. Averaged RTs and error rates were submitted to ANOVAs (task and distractor condition as variables) and subsequent $t$-tests testing for the effect of distractor condition per task. For the $t$-tests, we additionally computed Bayes factors $\left(\mathrm{BF}_{10}\right)$ with the BayesFactor package (Morey \& Rouder, 2015; default prior). Table 1 presents mean RTs and error rates. 
Table 1 Mean reaction times (RTs) (in ms) and error rates (\%) per task and distractor condition. Standard errors are given in parentheses

\begin{tabular}{lccccc}
\hline Distractor condition & \multicolumn{2}{l}{ Naming } & & \multicolumn{2}{l}{ Size decision } \\
\cline { 2 - 3 } \cline { 6 - 7 } & $M$ & $\%$ & & $M$ & $\%$ \\
\hline Related & $911(21)$ & $3.8(0.7)$ & $593(15)$ & $2.2(0.5)$ \\
Unrelated & $852(19)$ & $1.6(0.4)$ & $608(18)$ & $1.6(0.3)$ \\
Difference & $59(9)$ & $2.2(0.5)$ & $-15(6)$ & $0.6(0.4)$ \\
$95 \%$ CI & {$[41 ; 77]$} & {$[1.2 ; 3.2]$} & {$[-27 ;-3]$} & {$[-0.2 ; 1.4]$} \\
\hline
\end{tabular}

Note. $C I$ confidence interval. SEs and CIs based on cell means by participants

Responses were faster in size decision than in naming, $F_{1}(1,62)=120.48, p<.001, \eta_{\mathrm{g}}{ }^{2}=.650, F_{2}(1,39)=535.94$, $p<.001, \eta_{\mathrm{g}}{ }^{2}=.816$. Distractor condition was significant, $F_{1}(1,62)=14.94, p<.001, \eta_{\mathrm{g}}^{2}=.011, F_{2}(1,39)=8.77, p$ $=.005, \eta_{\mathrm{g}}{ }^{2}=.029$, as was the interaction of task and distractor condition, $F_{1}(1,62)=43.98, p<.001, \eta_{\mathrm{g}}^{2}=.032, F_{2}(1,39)=$ $27.40, p<.001, \eta_{\mathrm{g}}^{2}=.078$. In naming, related distractors slowed down responses, $t_{1}(31)=6.26, p<.001, d_{\mathrm{z}}=1.107$, $t_{2}(39)=4.40, p<.001, d_{\mathrm{z}}=0.696\left(\mathrm{BF}_{10}>200\right.$ for participants and items), whereas in size decision, they facilitated responses, $t_{1}(31)=2.54, p=.016, d_{\mathrm{z}}=0.449, t_{2}(39)=2.75, p$ $=.009, d_{\mathrm{z}}=0.435\left(\mathrm{BF}_{10}=2.91\right.$ for participants, $\mathrm{BF}_{10}=4.47$ for items).

In the errors, there was no effect of task, $F_{1}(1,62)=2.09, p$ $=.153, \eta_{\mathrm{g}}{ }^{2}=.024, F_{2}(1,39)=1.36, p=.250, \eta_{\mathrm{g}}{ }^{2}=.013$, but distractor condition was significant, $F_{1}(1,62)=17.29, p<$ $.001, \eta_{\mathrm{g}}{ }^{2}=.071, F_{2}(1,39)=9.22, p=.004, \eta_{\mathrm{g}}^{2}=.038$, as was the interaction of task and distractor condition, $F_{1}(1,62)=$ 5.57, $p=.021, \eta_{\mathrm{g}}^{2}=.024, F_{2}(1,39)=5.38, p=.026, \eta_{\mathrm{g}}{ }^{2}=$ .013. In naming, related distractors led to more errors, $t_{1}(31)=$ $4.18, p<.001, d_{\mathrm{z}}=0.738, t_{2}(39)=3.16, p=.003, d_{\mathrm{z}}=0.500$ $\left(\mathrm{BF}_{10}>10\right.$ for participants and items), but in size decision this was not the case, $t_{1}(31)=1.44, p=.161, d_{\mathrm{z}}=0.254, t_{2}(39)=$ $1.43, p=.160, d_{\mathrm{z}}=0.227\left(\mathrm{BF}_{10}=0.48\right.$ for participants, $\mathrm{BF}_{10}=$ 0.44 for items).

\section{Discussion}

We re-explored the locus of semantic interference in the PWI task by contrasting two task versions implemented in as parallel a fashion as possible but differing with respect to the processing stages involved: picture naming (requiring conceptual processing and lexical processing) and natural-size decision (requiring conceptual processing only). First, responses were much faster in size decision than in picture naming, ruling out that implicit naming was involved in size decision. Second, we obtained semantic interference in picture naming, replicating the standard effect and demonstrating the sensitivity of our materials and procedure. Third, an effect was also observed in size decision, but - critically - with different polarity. That is, we observed semantic interference in naming and semantic facilitation in size decision. This pattern rules out that part of the semantic interference effect is located at the conceptual level and is compatible with a lexical locus view (e.g., Abdel Rahman \& Melinger, 2009; Damian \& Bowers, 2003; Levelt et al., 1999; Roelofs, 1992; Schriefers et al., 1990). Furthermore, the polarity shift we observed supports the idea that semantic interference in PWI reflects the net effect of facilitation during conceptual processing (due to priming) and interference during lexical processing (due to competitive selection; Abdel Rahman \& Melinger, 2009; Hantsch et al., 2009; Navarrete \& Costa, 2005).

As an alternative to the competitive lexical selection view, some authors have argued that semantic interference in PWI might be a marker of a task-specific control mechanism operating at the post-lexical level on an articulatory output buffer that comes into play whenever word distractors are used (response-exclusion hypothesis, Finkbeiner \& Caramazza, 2006; Mahon, Costa, Peterson, Vargas, \& Caramazza, 2007). Although the present data do not rule out such an account, much independent empirical evidence has accumulated that, on balance, seems to be more compatible with the lexical view (e.g., Aristei, Melinger, \& Abdel Rahman, 2011; Piai, Roelofs, \& Schriefers, 2011; Vieth, McMahon, \& de Zubicaray, 2014). For example, semantic interference is also obtained from distractor words in task configurations requiring no articulation (Abdel Rahman \& Aristei, 2010), from distractor pictures (Mädebach et al., 2018b), or from distractor sounds (Mädebach, Wöhner, Kieseler, \& Jescheniak, 2017; Mädebach, Kieseler, \& Jescheniak, 2018a).

The pattern we found also converges with a recent result obtained with a different prominent speech production task, blocked-cyclic naming. In this task pictures are either presented blocked by semantic category (homogeneous context) or intermixed (heterogeneous context) over a number of cycles (repetitions of pictures). Typically, responses are slowed down in the homogeneous context from the second cycle onwards. Using this task, Belke (2013) compared semantic picture classification (living vs. non-living) with picture naming and observed effects of semantic context with different polarity: facilitation in semantic classification and interference in naming. From the presence of semantic facilitation in classification, Belke concluded that the conceptual level is involved in but cannot be the locus of semantic interference found in picture naming. She suggested that the overall pattern of results is best explained by assuming that semantic interference in blocked-cyclic picture naming has a conceptual origin and lexical locus. The term origin denotes the processing level at which a change underlying a respective effect occurs and the term locus refers to the processing level at which the behavioral consequence arises. The finding presented here suggests that the same holds true for semantic interference in the PWI 
task. The origin of the effect seems to be at the conceptual level, likely due to mutual priming of distractor and target (leading to facilitation in size decision), while its locus seems to be at the lexical level, likely due to competitive lexical selection (leading to interference in naming), supporting a parsimonious account of semantic context effects obtained with different speech production tasks (Roelofs, 2018).

One caveat regarding the task contrast implemented in our study (and all similar studies) should be mentioned. The study's logic relies on the idea that the lexical and nonlexical task demanded processing at the shared conceptual level. To be clear, we do not wish to claim that exactly the same conceptual information was activated in lexical retrieval and in size decision. But we wish to claim that our non-lexical task demanded conceptual activation to a degree sufficient for detecting some trace of interference, should it exist at that level. The finding of a distractor effect in size decision suggests that this was likely the case.

\section{Conclusion}

Our study supports the view that semantic interference in PWI has its locus at the lexical level and that the conceptual level is only its origin, not an additional locus. Other than in earlier studies (Damian \& Bowers, 2003; Schriefers et al., 1990), this conclusion is based on positive evidence enabling us to localize an effect central to theory building with greater confidence than possible so far.

Author Note This work was supported by the German Research Council (DFG, grant number JE229/12-2). We thank Andreas Mädebach, Herbert Schriefers, and two anonymous reviewers for helpful comments on a previous version of this article.

\section{References}

Abdel Rahman, R., \& Aristei, S. (2010). Now you see it ... and now again: Semantic interference reflects lexical competition in speech production with and without articulation. Psychonomic Bulletin \& Review, 17, 657-661. https://doi.org/10.3758/PBR.17.5.657

Abdel Rahman, R., \& Melinger, A. (2009). Semantic context effects in language production: A swinging lexical network proposal and a review. Language and Cognitive Processes, 24, 713-734. https:// doi.org/10.1080/01690960802597250

Aristei, S., Melinger, A., \& Abdel Rahman, R. (2011). Electrophysiological chronometry of semantic context effects in language production. Journal of Cognitive Neuroscience, 23, 15671586. https://doi.org/10.1162/jocn.2010.21474

Belke, E. (2013). Long-lasting inhibitory semantic context effects on object naming are necessarily conceptually mediated. Implications for models of lexical-semantic encoding. Journal of Memory and Language, 69, 228-256. https://doi.org/10.1016/j.jml.2013.05.008

Bloem, I., \& La Heij, W. (2003). Semantic facilitation and semantic interference in word translation: Implications for models of lexical access in language production. Journal of Memory and Language, 48, 468-488. https://doi.org/10.1016/S0749-596X(02)00503-X

Collins, A. M., \& Loftus, E. F. (1975). A spreading-activation theory of semantic processing. Psychological Review, 82, 407-428. https:// doi.org/10.1037/0033-295X.82.6.407

Damian, M. F., \& Bowers, J. S. (2003). Locus of semantic interference in picture-word interference tasks. Psychonomic Bulletin \& Review, 10, 111-117. https://doi.org/10.3758/BF03196474

Dell, G. S., Martin, N., \& Schwartz, M. F. (2007). A case-series test of the interactive two-step model of lexical access: Predicting word repetition from picture naming. Journal of Memory and Language, 56, 490-520. https://doi.org/10.1016/j.jml.2006.05.007

Dell'Acqua, R., Job, R., Peressotti, F., \& Pascali, A. (2007). The pictureword interference effect is not a Stroop effect. Psychonomic Bulletin \& Review, 14, 717-722.https://doi.org/10.3758/BF03196827

Finkbeiner, M., \& Caramazza, A. (2006). Now you see it, now you don't: On turning semantic interference into facilitation in a Stroop-like task. Cortex: A Journal Devoted to the Study of the Nervous System and Behavior, 42, 790-796. https://doi.org/10.1016/S00109452(08)70419-2

Glaser, W. R., \& Düngelhoff, F. J. (1984). The time course of pictureword interference. Journal of Experimental Psychology: Human Perception and Performance, 10, 640-654. https://doi.org/10.1037/ 0096-1523.10.5.640

Hantsch, A., Jescheniak, J. D., \& Schriefers, H. (2005). Semantic competition between hierarchically related words during speech planning. Memory \& Cognition, 33, 984-1000. https://doi.org/10.3758/ BF03193207

Hantsch, A., Jescheniak, J. D., \& Schriefers, H. (2009). Distractor modality can turn semantic interference into semantic facilitation in the picture-word interference task: Implications for theories of lexical access in speech production. Journal of Experimental Psychology: Learning, Memory, and Cognition, 35, 1443-1453. https://doi.org/ 10.1037/a0017020

Jescheniak, J. D., Matushanskaya, A., Mädebach, A., \& Müller, M. M. (2014). Semantic interference from distractor pictures in singlepicture naming: Evidence for competitive lexical selection. Psychonomic Bulletin \& Review, 21, 1294-1300. https://doi.org/10. 3758/s13423-014-0606-5

Jescheniak, J. D., Oppermann, F., Hantsch, A., Wagner, V., Mädebach, A., \& Schriefers, H. (2009). Do perceived context pictures automatically activate their phonological code? Experimental Psychology, 56, 56-65. https://doi.org/10.1027/1618-3169.56.1.56

Jescheniak, J. D., Schriefers, H., \& Hantsch, A. (2003). Utterance format effects phonological priming in the picture-word task: Implications for models of phonological encoding in speech production. Journal of Experimental Psychology: Human Perception and Performance, 29, 441-454. https://doi.org/10.1037/0096-1523.29.2.441

Kuipers, J.-R., \& La Heij, W. (2008). Semantic facilitation in category and action naming: Testing the message-congruency account. Journal of Memory and Language, 58, 123-139. https://doi.org/10. 1016/j.jml.2007.05.005

La Heij, W. (1988). Components of Stroop-like interference in picture naming. Memory \& Cognition, 16, 400-410. https://doi.org/10. 3758/BF03214220

Lambon Ralph, M. A. (2014). Neurocognitive insights on conceptual knowledge and its breakdown. Philosophical transactions of the Royal Society of London. Series B, Biological sciences, 369, 20120392. https://doi.org/10.1098/rstb.2012.0392

Levelt, W. J. M., Roelofs, A., \& Meyer, A. S. (1999). A theory of lexical access in speech production. Behavioral and Brain Sciences, 22, 175. https://doi.org/10.1017/S0140525X99001776 
Lupker, S. J. (1979). The semantic nature of response competition in the picture-word interference task. Memory \& Cognition, 7, 485-495. https://doi.org/10.3758/BF03198265

Lupker, S. J., \& Katz, A. N. (1981). Input, decision, and response factors in picture-word interference. Journal of Experimental Psychology: Human Learning and Memory, 7, 269-282. https://doi.org/10.1037// 0278-7393.7.4.269

Mädebach, A., Kieseler, M.-L., \& Jescheniak, J. D. (2018a). Localizing semantic interference from distractor sounds in picture naming: A dual-task study. Psychonomic Bulletin \& Review, 25, 1909-1916. https://doi.org/10.3758/s13423-017-1386-5

Mädebach, A., Wöhner, S., \& Jescheniak, J. D. (2018b). Opening a file drawer - Surprisingly robust evidence for semantic interference from distractor pictures in picture naming. Poster presented at the International Workshop on Language Production 2018, Nijmegen, The Netherlands. https://osf.io/r8d92/

Mädebach, A., Wöhner, S., Kieseler, M.-L., \& Jescheniak, J. D. (2017). Neighing, barking, and drumming horses - Object related sounds help and hinder picture naming. Journal of Experimental Psychology: Human Perception and Performance, 43, 1629-1646. https://oi.org/10.1037/xhp0000415

Mahon, B. Z., Costa, A., Peterson, R., Vargas, K. A., \& Caramazza, A. (2007). Lexical selection is not by competition: A reinterpretation of semantic interference and facilitation effects in the picture-word interference paradigm. Journal of Experimental Psychology: Learning, Memory, and Cognition, 33, 503-535. https://doi.org/10. 1037/0278-7393.33.3.503

Matushanskaya, A., Mädebach, A., Müller, M. M., \& Jescheniak, J. D. (2016). When sufficiently processed, semantically related distractor pictures hamper picture naming. Experimental Psychology, 63, 307317. https://doi.org/10.1027/1618-3169/a000340

Meyer, A. S. (1996). Lexical access in phrase and sentence production: Results from picture-word interference experiments. Journal of Memory and Language, 35, 477-496. doi:https://doi.org/10.1006/ jmla.1996.0026

Morey, R. D., \& Rouder, J. N. (2015). BayesFactor 0.9.12-2. Comprehensive R Archive Network. http://cran.r-project.org/web/ packages/BayesFactor/index.html

Morsella, E., \& Miozzo, M. (2002). Evidence for a cascade model of lexical access in speech production. Journal of Experimental Psychology: Learning, Memory, and Cognition, 28, 555-563. https://doi.org/10.1037//0278-7393.28.3.555

Navarrete, E., \& Costa, A. (2005). Phonological activation of ignored pictures: Further evidence for a cascade model of lexical access. Journal of Memory and Language, 53, 359-377. https://doi.org/10. 1016/j.jml.2005.05.001
Navarrete, E., Del Prato, P., Peressotti, F., \& Mahon, B. Z. (2014). Lexical selection is not by competition: Evidence from the blocked naming paradigm. Journal of Memory and Language, 76, 253-272. https:// doi.org/10.1016/j.jml.2014.05.003

Oppenheim, G. M., Dell, G. S., \& Schwartz, M. F. (2010). The dark side of incremental learning: A model of cumulative semantic interference during lexical access in speech production. Cognition, 114, 227-252. https://doi.org/10.1016/j.cognition.2009.09.007

Piai, V., Roelofs, A., \& Schriefers, H. (2011). Semantic interference in immediate and delayed naming and reading: Attention and task decisions. Journal of Memory and Language, 64, 404-423. https:// doi.org/10.1016/j.jml.2011.01.004

Piai, V., Roelofs, A., \& Schriefers, H. (2014). Locus of semantic interference in picture naming: Evidence from dual-task performance. Journal of Experimental Psychology: Learning, Memory, and Cognition, 40, 147-165. https://doi.org/10.1037/a0033745

Roelofs, A. (1992). A spreading-activation theory of lemma retrieval in speaking. Cognition, 42, 107-142. https://doi.org/10.1016/00100277(92)90041-F

Roelofs, A. (2008). Tracing attention and the activation flow of spoken word planning using eye movements. Journal of Experimental Psychology: Learning, Memory, and Cognition, 34, 353-368. https://doi.org/10.1037/0278-7393.34.2.353

Roelofs, A. (2018). A unified computational account of cumulative semantic, semantic blocking, and semantic distractor effects in picture naming. Cognition, 172, 59-72. https://doi.org/10.1016/j.cognition. 2017.12.007

Schriefers, H., Meyer, A. S., \& Levelt, W. J. M. (1990). Exploring the time course of lexical access in language production: Picture-word interference studies. Journal of Memory and Language, 29, 86-102. https://doi.org/10.1016/0749-596X(90)90011-N

Starreveld, P. A., \& La Heij, W. (1995). Semantic interference, orthographic facilitation, and their interaction in naming tasks. Journal of Experimental Psychology: Learning, Memory, and Cognition, 21, 686-698. https://doi.org/10.1037/0278-7393.21.3.686

Van Casteren, M., \& Davis, M. H. (2006). Mix, a program for pseudorandomization. Behavior Research Methods, 38, 584-589. https://doi.org/10.3758/BF03193889

Vieth, H. E., McMahon, K. L., \& de Zubicaray, G. I. (2014). Feature overlap slows lexical selection: Evidence from the picture-word interference paradigm. The Quarterly Journal of Experimental Psychology, 67, 2325-2339. https://doi.org/10.1080/17470218. 2014.923922

Publisher's note Springer Nature remains neutral with regard to jurisdictional claims in published maps and institutional affiliations. 